Das Buch liefert ein facettenreiches, abgewogenes, gelungenes Portrait der Linken, dessen Lektüre auf jeden Fall lohnt. Auch mit seinem umfangreichen statistischen Material zur Wähler- und Mitgliedschaft der PDS/Linke hat es als Nachschlagwerk seinen Wert. Zugleich werden interne Dokumente präsentiert, die Einblicke in die ideologische Selbstbeschreibung und in Richtungskonflikte geben. Mit über 20 Seiten Literaturverzeichnis greift der Band die wesentlichen Befunde und Erkenntnisse des angeschwollenen Literaturbestands über Die Linke auf und liefert der weiteren Forschung, womöglich im Disput, wichtige Anregungen.

Elmar Wiesendahl

\title{
Linksextremismus im europäischen Vergleich: methodisch aufwendige, theoretisch zu enge Studie
}

Mannewitz, Tom: Linksextremistische Parteien in Europa nach 1990. Ursachen für Wablerfolge und-misserfolge (Reihe Extremismus und Demokratie, Bd. 23), Nomos Verlagsgesellschaft, Baden-Baden 2012, 506 Seiten, $€ 74,-$.

In den letzten 30 Jahren hat sich die vergleichende Wahl- und Parteienforschung intensiv mit den neuen oder erneuerten Parteien am rechten Rand beschäftigt, die vor allem seit den 1980er Jahren in zahlreichen europäischen Ländern entstanden sind. Die Entwicklungen am linken Rand des Parteienspektrums sind darüber oft in Vergessenheit geraten. Tom Mannewitz hat sich in seiner Chemnitzer Dissertation vorgenommen, diese Lücke zumindest teilweise zu schließen, indem er für den Zeitraum seit 1990 die internen und externen Bedingungen für den Erfolg linker Flügelparteien untersucht. Der Epochenbruch von 1990 ist dabei für den Gegenstand in doppelter Weise bedeutsam: Zum einen eröffnet sich für den Parteienvergleich erst durch die Demokratisierung in Mittel- und Osteuropa eine sinnvolle Perspektive. Zum anderen hat der Untergang des Staatssozialismus in Europa auch in den westlichen Ländern die Bedingungen, unter denen Parteien am linken Rand operieren, radikal verändert.

Das Panorama, das Mannewitz entfaltet, ist auf den ersten Blick beeindruckend: Behandelt werden 21 Parteien beziehungsweise Wahlbündnisse in zehn europäischen Ländern (Belgien, Dänemark, Deutschland, Frankreich, Italien, Österreich, Polen, Schweiz, Slowakei und Tschechien). Ob diese Gruppierungen jedoch jeweils in ihrer Gänze als „linksextremistisch“ bezeichnet werden können, erscheint im Falle der deutschen Linkspartei, aber auch der italienischen PRC, der PdCI und des Linksbündnisses „La Sinistra L'Arcobaleno" diskussionswürdig. Dementsprechend vermeidet Mannewwitz zumindest im Fall der Linkspartei in den entsprechenden Textpassagen den Begriff des Extremismus und spricht statt dessen von einer „Linksaußenpartei“, ohne das Problem, dass sich daraus für seine Studie ergibt, zu thematisieren.

Neben Einleitung und Schlussbetrachtung umfasst die Arbeit acht inhaltliche Kapitel. Nach einer konzisen Darstellung der verwendeten Begrifflichkeit und der Kriterien für die Fallauswahl (Kapitel 2) nimmt die fast lehrbuchartige Darstellung der in der Arbeit verwendeten Methode (QCA, Kapitel 3) mit rund fünfzig Seiten ungewöhnlich breiten Raum 
ein, während die Herleitung forschungsleitender Hypothesen in Kapitel 4 vergleichsweise knapp ausfällt und sich im Wesentlichen auf die Auflistung potentiell relevanter Kontextfaktoren beschränkt.

Das umfangreiche fünfte Kapitel, das fast ein Drittel der Arbeit ausmacht, stellt - gegliedert nach Ländern - die 21 Parteien beziehungsweise Parteienbündnisse in ihrem politischen Kontext dar. Dabei fällt auf, dass sich der Autor in weiten Teilen auf deutschsprachige Sekundärliteratur stützt, während der Umfang der berücksichtigten internationalen Forschungsliteratur für eine europäisch-vergleichende Studie hier wie in den anderen Kapiteln eher gering erscheint. In Kapitel sechs, sieben und acht versucht Mannewitz dann mit Hilfe der QCA Regelmäßigkeiten in den Wahlerfolgen beziehungsweise -misserfolgen der linken Flügelparteien aufzudecken. Interessant ist vor allem, dass Linksparteien dann elektoral besonders erfolgreich zu sein scheinen, wenn die Arbeitslosigkeit hoch ist und wenn sie sich nicht an der Regierung beteiligen (beziehungsweise nicht beteiligt werden). Ersteres steht im Einklang mit der Klientelhypothese, letzteres deutet darauf hin, dass die im $\mathrm{Zu}$ sammenhang mit nicht-etablierten Parteien häufig diskutierte Strategie der „Entzauberung“ zumindest in den hier betrachteten Fällen wirksam gewesen sein könnte. Zwei weitere Befunde - die Linksparteien erzielen dann gute Resultate, wenn es nicht zu Spaltungen kommt beziehungsweise wenn andere Parteien stark sind - erscheinen hingegen recht trivial, da sie sich leicht auf die Nullsummen-Natur von Wahlen zurückführen lassen. Im vorletzten Kapitel lotet Mannewitz Möglichkeiten der Generalisierung seiner Befunde aus, im zehnten Kapitel fasst er die Hauptergebnisse noch einmal zusammen.

Obwohl der Autor sich um eine explizite theoretische Fundierung bemüht, hinterlässt das Buch letztlich einen unbefriedigenden Eindruck. Die Gründe hierfür liegen auf der Hand: Mannewitz' Arbeit ist zwar extremismustheoretisch aufgeladen, vermeidet aber die Auseinandersetzung mit der Frage, in welchem Umfang die hier betrachteten Parteien tatsächlich gegen die liberale Demokratie gerichtet sind und inwieweit die Extremismustheorie ihre Wahl erklären kann. Auch andere Theorien aus dem Bereich des Wählerverhaltens, der Parteiensoziologie und des Parteienwettbewerbs finden kaum Berücksichtigung. Die unterschiedlichen Eigenschaften der Parteien spielen nur eine geringe Rolle, über die Motive ihrer (zahlenmäßig oft geringen) Wählerschaft kann aufgrund der berücksichtigen Daten nur spekuliert werden. Trotz des großen Aufwandes, der mit der QCA getrieben wird, bleibt der Leser deshalb am Ende des Buches etwas ratlos zurück. 